\title{
A SPECTROSCOPIC SEARCH FOR HOT (B-TYPE) POST-AGB STARS
}

\author{
E.S. CONLON \\ Department of Pure and Applied Physics, The Queen's University of Belfast, Belfast BT7 1NN, \\ Northern Ireland
}

\begin{abstract}
At Queen's University, we have been undertaking a spectroscopic programme to elucidate the nature of faint blue stars at high galactic latitudes. We have identified approximately 50 that appear to spectroscopically identical (even at high resolution and signal-to-noise) to normal young Population I B-type stars in the galactic disc. However, we have also found seven faint objects () that were previously classified as Population I on the basis of photometry and/or low resolution spectroscopy; careful model atmosphere analyses of high resolution spectra now indicate that they have non-Population I compositions. Their derived atmospheric parameters are coincident with theoretical post-AGB evolutionary tracks and thus together with their peculiar composition, they would appear to be hot evolved post-AGB objects.
\end{abstract}

A systematic search for other B-type post-AGB objects has been initiated. Criteria for target selection include high latitude, supergiant spectral classification and characteristic post-AGB infrared features. Spectroscopic data have been obtained at the Anglo-Australian Telescope and Mount Stromlo Observatory and analysed using model atmosphere techniques. Preliminary results are presented here. 